\title{
Silver/Dendrimer Nanocomposites as Biomarkers: Fabrication, Characterization, In Vitro Toxicity and Intracellular Detection
}

\author{
Wojciech Lesniak ${ }^{1,2, *}$, Anna U. Bielinska ${ }^{1}$, Kai Sun $^{3}$, Katarzyna W Janczak ${ }^{1}$, Xiangyang Shi ${ }^{1}$, \\ James R. Baker Jr. ${ }^{1}$, and Lajos P. Balogh ${ }^{1,4,5, *}$ \\ ${ }^{1}$ Center for Biologic Nanotechnology, ${ }^{2}$ Microscopy and Image-Analysis Laboratory, ${ }^{3}$ Electron \\ Microbeam Analysis Laboratory, ${ }^{4}$ Department of Biomedical Engineering, ${ }^{5}$ Macromolecular \\ Science and Engineering, University of Michigan, Ann Arbor, MI 48109
}

\begin{abstract}
Abbreviations:
PAMAM_E5. $\mathrm{NH}_{2}$ - amine-terminated, ethylenediamine core generation 5 poly(amidoamine) dendrimer

PAMAM_E5.NSAH - succinamic acid derivative of PAMAM_E5. $\mathrm{NH}_{2}$ dendrimer PAMAM_E5.NGly - glycidol derivative of PAMAM_E5. $\mathrm{NH}_{2}$ dendrimer $\left[\left(\mathrm{Ag}^{+}\right)_{25}\right.$-PAMAM_E5.NH $\left.{ }_{2}\right]$ and $\left\{\left(\mathrm{Ag}^{0}\right)_{25}\right.$-PAMAM_E5.NH $\left.\mathrm{NH}_{2}\right\}$ - silver complex and silver nanocomposite of PAMAM_E5.NH $\mathrm{NH}_{2}$ dendrimer containing twenty-five silver per dendrimer

$\left[\left(\mathrm{Ag}^{+}\right)_{25}\right.$-PAMAM_E5.NGly] and $\left\{\left(\mathrm{Ag}^{0}\right)_{25}\right.$-PAMAM_E5.NGly $\}$ - silver complex and silver nanocomposite of PAMAM_E5.NGly dendrimer

$\left[\left(\mathrm{Ag}^{+}\right)_{25}\right.$-PAMAM_E5.NSAH $]$ and $\left\{\left(\mathrm{Ag}^{0}\right)_{25}\right.$-PAMAM_E5.NSAH $\}$ - silver complex and silver nanocomposite of $\mathrm{P}$ AMAM_E5.NSAH dendrimer

DNC - dendrimer nanocomposite

DLS - dynamic light scattering

HRTEM - high-resolution transmission electron microscopy

EDS - X-ray energy dispersive spectroscopy

SAED - selected area electron diffraction

DIC - differential interference contrast

*: Present address: Roswell Park Cancer Institute, Elm and Carlton Streets, Buffalo, NY 14263

Ph: 716-845-3886, F: 716-845-8254; Email: lajos.balogh@roswellpark.org Supporting material
\end{abstract}

Materials. EDA-core poly(amidoamine) PAMAM dendrimer of generation 5 (PAMAM_E5. $\mathrm{NH}_{2}$ ) was purchased from Dendritech Midland, MI, in methanol solution (14.17 wt\%). Succinic 
anhydride, glycidol, absolute methanol, dimethylsulfoxide (DMSO), silver nitrate, and all the other chemicals and solvents were obtained from Aldrich and used as received. Regenerated cellulose dialysis membranes $(\mathrm{MWCO}=10,000)$ were acquired from Fisher. Water used in the experiments was purified by a Milli-Q Plus 185 water purification system (Millipore, Bedford, MA, USA) with

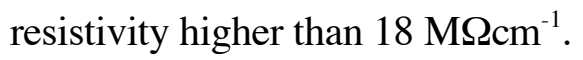

Template preparation. The dry form of PAMAM_E5. $\mathrm{NH}_{2}$ dendrimer was obtained by evaporation of methanol on a rotary evaporator followed by lyophilization from aqueous solution. The resulting white solids were used in further studies without additional purification. PAMAM succinamic acid, PAMAM_E5.NHCOCH${ }_{2} \mathrm{CH}_{2} \mathrm{COOH}$ (abbreviated as PAMAM_E5.NSAH) and PAMAM glycidol derivative, PAMAM_E5.N $\left(\mathrm{CH}_{2} \mathrm{CHOHCH}_{2} \mathrm{OH}\right)_{2}$ (abbreviated as PAMAM_E5.NGly) dendrimers were synthesized according to previously reported procedures. ${ }^{1,2}$

Silver/dendrimer nanocomposite preparation. (1) Silver binding: In order to examine the binding capacity of PAMAM_E5. $\mathrm{NH}_{2}$ towards $\mathrm{Ag}^{+}$ions, the complexation of $\mathrm{Ag}^{+}$to dendrimer was examined by potentiometric titration. Titrations were performed over a $\mathrm{pH}$ range of $2.5-12$, at room temperature, under nitrogen atmosphere. Concentration of dendrimer was $3.7 \times 10^{-5} \mathrm{M}$, and the silver to dendrimer molar ratios were set to $15: 1,30: 1$, and 45:1. $\mathrm{AgNO}_{3}$ was used to prepare a stock solution of $\mathrm{Ag}^{+}$ions $\left(5.78 \times 10^{-2} \mathrm{M}\right)$. Dendrimer was dissolved in the $0.1 \mathrm{M} \mathrm{NaNO}_{3}$ solution and the $\mathrm{pH}$ was adjusted to 2.5 by $\mathrm{HNO}_{3}(1.51 \mathrm{M})$. Samples were prepared by mixing appropriate volumes of stock solutions of dendrimer and $\mathrm{AgNO}_{3}$ to reach a final volume of $8.5 \mathrm{~mL}$. Titrations were performed using $0.1021 \mathrm{M} \mathrm{NaOH}$ standard solution.

(2) Nanocomposite fabrication: Based on the potentiometric data, synthesis of silver nanocomposites with PAMAM_E5.NH, PAMAM_E5.NGly, and PAMAM_E5.NSAH dendrimers was designed as follows. In each case $10 \mathrm{mg}$ of dendrimer was dissolved in $8.5 \mathrm{~mL}$ of water and $\mathrm{pH}$ was adjusted to 2.5 by adding sufficient amount of $\mathrm{HNO}_{3}(1.53 \mathrm{M})$ with vigorous stirring. A calculated amount of $\mathrm{AgNO}_{3}\left(1.1 \times 10^{-1} \mathrm{M}\right)$ stock solution was subsequently added dropwise to obtain the $\mathrm{Ag}^{+}$: dendrimer 25:1 molar ratio. The $\mathrm{Ag}^{+}$: dendrimer molar ratio was calculated based on average molecular weight of PAMAM_E5.NH $\mathrm{NH}_{2}$ - 28726, PAMAM_E5.NGly - 
39590 and PAMAM_E5.NSAH - 41639 dendrimer templates, obtained from size exclusion chromatography. This was followed by titration using $0.1021 \mathrm{M} \mathrm{NaOH}$ solution, which was stopped at $\mathrm{pH}$ 7.4. The final concentration of dendrimer template was $1 \mathrm{mg} / \mathrm{mL}$. To minimize chemical contamination of the samples, the resulting solutions of $\left[\left(\mathrm{Ag}^{+}\right)_{25}-\mathrm{PAMAM}\right.$ E5.NH $\left.{ }_{2}\right]$, $\left[\left(\mathrm{Ag}^{+}\right)_{25}\right.$-PAMAM_E5.NGly], and $\left[\left(\mathrm{Ag}^{+}\right)_{25}\right.$-PAMAM_E5.NSAH] complexes were exposed to intense (30w) UV irradiation $\left(340 \mathrm{~nm}\right.$ ) for $12 \mathrm{~h}$ to promote the photolytic reduction of $\mathrm{Ag}^{+}$cations to $\mathrm{Ag}^{0}$.

Spectroscopy. Aqueous solutions of $\left\{\left(\mathrm{Ag}^{0}\right)_{25}\right.$-PAMAM_E5.NH $\left.\mathrm{NH}_{2}\right\},\left\{\left(\mathrm{Ag}^{0}\right)_{25}\right.$-PAMAM_E5.NGly $\}$, and $\left\{\left(\mathrm{Ag}^{0}\right)_{25}\right.$-PAMAM_E5.NSAH $\}$ nanocomposites were studied by means of UV-Vis and fluorescence spectroscopy, dynamic light scattering, and zeta potential analysis. UV-Vis spectra were obtained using a Perkin-Elmer Lambda $20 \mathrm{UV}$-Vis spectrophotometer over the spectral range of 190-1000 nm in $1 \mathrm{~cm}$ quartz cuvettes. Fluorescence spectra were measured using a Fluoromax 3 spectrofluorometer and $1 \mathrm{~cm}$ quartz cuvette. Dynamic light scattering and zeta potential measurements were conducted using a NICOMP 380/ZLS Zeta Potential Analyzer.

Cell lines: KB human epithelial, Rat2 embryonic fibroblast, U937 human monocytes and mouse NIH3T3 embryonic fibroblast cell lines were used in the biological studies.

Biocompatibility testing. Cytotoxicity of dendrimers and related silver nanocomposites was measured using XTT colorimetric assay of cellular viability (Roche Diagnostic, cat. No.: 1465 015). Briefly, $5 \times 10^{3}$ to $1 \times 10^{4} \mathrm{~KB}$ human epithelial cells per well were seeded into a 96 well plate. After overnight incubation, the culture medium (RPMI1640, 10\% FBS) was removed and $100 \mu$ of silver DNC or its parental dendrimer at concentrations ranging from $1 \mathrm{nM}$ to $2 \mu \mathrm{M}$ in PBS $\mathrm{pH} 7.4$ was added. After $2 \mathrm{~h}$ incubation at $37 \mathrm{C}$, reagent solutions were removed and $100 \mu \mathrm{L}$ of XTT reagent in PBS was added. The assays were carried out according to the manufacturer's instructions. Absorbance measurements were performed using a Spectra Max 340 ELISA reader (Molecular Devices, Sunnyvale, CA) at $492 \mathrm{~nm}$ and the reference wavelength of $690 \mathrm{~nm}$. The same assay for Rat2, NIH3T3 and U937 cell lines. 
Confocal microscopy analysis. Rat2 embryonic fibroblast cell line was seeded at a density of $5 \mathrm{x}$ $10^{4}$ cells $/ \mathrm{cm}^{2}$ in RPMI 1640, 10\% FBS on the glass-bottom microwell dishes no.1.5 (MatTek Corp. cat no. P350) and incubated overnight at 37 C. After removal of culture medium, the Rat2 cells were incubated with dendrimer templates or silver DNCs at concentrations of $100 \mathrm{nM}, 500 \mathrm{nM}$ and $1 \mu \mathrm{M}$ in PBS $\mathrm{pH} 7.4$ for $2 \mathrm{~h}$ at $37 \mathrm{C}$. For microscopic analysis the reagents were removed, cells were washed 5 times with PBS and fixed with 2\% formaldehyde in PBS. Cell uptake studies were conducted using Zeiss LSM 510 confocal microscope equipped with UV laser operating at $364 \mathrm{~nm}$ and an emission filter that transmits light between 465 and $485 \mathrm{~nm}$.

The morphology, composition and crystallites of nanoparticles were analyzed by means of highresolution transmission electron microscopy, X-ray energy dispersive spectroscopy and selected area electron diffraction, respectively, using a JEOL 2010F analytical TEM operated at $200 \mathrm{kV}$. Specimens were prepared by mounting a drop of aqueous solution of nanoparticles on carboncoated copper grids and allowing it to dry in air. In order to avoid damage of biologic samples, the cell cultures were examined by a Phillips CM 100 TEM microscope operating at $60 \mathrm{kV}$. Images were recorded using Hamamatsu digital camera, controlled by AMT (advance microscopy technology) software. The specimens were prepared according to the following procedures. The human U937 monocytes and NIH3T3 mouse embryonic fibroblast cell lines were aliquoted in $5 \mathrm{~mL}$ tubes at a concentration of $1 \times 10^{6}$ cells $/ \mathrm{mL}$. After overnight growth at $37 \mathrm{C}$, the medium was removed and 2\% FBS solution containing $100 \mathrm{nM}$ or $500 \mathrm{nM}$ of nanocomposites was added, and the incubation was carried out for $1 \mathrm{~h}$ at $37 \mathrm{C}$. Then, medium was removed and cells were washed with Sorenson buffer and fixed at room temperature for $20 \mathrm{~min}$. using $2.5 \%$ of glutaraldehyde in PBS buffer, which was followed by addition of $0.1 \% \mathrm{NaBH}_{4}$ in PBS buffer. After 15 min. of quenching cells were rinsed 5 times with Sorenson buffer, resuspended in the same medium and post-fixed using osmium tetroxide for $1 \mathrm{~h}$. After additional washing in buffer cells were dehydrated in a series of ethanol solutions of 30\%, 50\%, 70\%, 90\% and 99.9\%. Samples were further infiltrated using the following sequence of mixtures of $99.9 \%$ ethanol and resin: 3 parts of $\mathrm{EtOH}+1$ part resin (for $1 \mathrm{~h}$ ), 1 parts of $\mathrm{EtOH}+1$ part resin (for $1 \mathrm{~h}$ ), 1 parts of $\mathrm{EtOH}+$ 
3 part resin (for overnight), full strength resin $(4 \mathrm{~h})$, full strength resin (overnight). After the third change of resin, polymerization was performed and $75 \mathrm{~nm}$ sections were obtained using a Reichart Ultramicroton. Sections were mounted on 200 mesh copper grids.

\section{References}

(1) Quintana, A.; Raczka, E.; Piehler, L.; Lee, I.; Myc, A.; Majoros, I.; Patri, A. K.; Thomas, T.; Mule, J.; Baker, J. R.; Pharm. Res. 2002, 19, 1310.

(2) Uppuluri, S.; Swanson, D. R.; Piehler, L. T.; Li, J.; Hagnauer, G. L.; Tomalia, D. A.; Adv. Mater. 2000, 12, 796. 


\section{Figures}

Figure 1s. UV-vis spectra of: $A-\left\{\left(\mathrm{Ag}^{0}\right)_{25}\right.$-PAMAM_E5.NGly $\}$ (solid line), exposed to UV PAMAM_E5.NGly (dotted line); B - $\left\{\left(\mathrm{Ag}^{0}\right)_{25}\right.$-PAMAM_E5.NH $\left.\mathrm{NH}_{2}\right\}$ (solid line), exposed to UV PAMAM_E5.NH $\mathrm{NH}_{2}$ (dotted line) and C $-\left\{\left(\mathrm{Ag}^{0}\right)_{25}\right.$-PAMAM_E5.NSAH $\}$ (solid line), exposed to UV PAMAM_E5.NSAH (dotted line) nanocomposites and dendrimer templates. Recorded for aqueous samples at dendrimer concentration of $1 \mathrm{mg} / \mathrm{mL}$ and $\mathrm{pH}$ 7.4.

Figure 2s TEM images of A - $\left\{\left(\mathrm{Ag}^{0}\right)_{25}\right.$-PAMAM_E5.NH $\}$; B - $\left\{\left(\mathrm{Ag}^{0}\right)_{25}\right.$-PAMAM_E5.NGly $\}$ and $\mathrm{C}-\left\{\left(\mathrm{Ag}^{0}\right)_{25}\right.$-PAMAM_E5.NSAH $\}$ nanoparticles on recorded at magnification of 92000x.

Figure 3s. A - High resolution TEM pattern; B - SAED images and C - EDS spectrum of $\left\{\left(\mathrm{Ag}^{0}\right)_{25}\right.$-PAMAM_E5.NH $\left.\mathrm{NH}_{2}\right\}$ nanocomposite (similar results were obtained for $\left\{\left(\mathrm{Ag}^{0}\right)_{25^{-}}\right.$ PAMAM_E5.NGly $\}$ and $\left\{\left(\mathrm{Ag}^{0}\right)_{25}\right.$-PAMAM_E5.NSAH $\}$.

Figure 4s. Excitation (A) and emission (B) fluorescence spectra of $\left\{\left(\mathrm{Ag}^{0}\right)_{25^{-}}\right.$ PAMAM_E5.NH $\}$, (solid line), $\left\{\left(\mathrm{Ag}^{0}\right)_{25}\right.$-PAMAM_E5.NGly $\}$ (dotted line), and $\left\{\left(\mathrm{Ag}^{0}\right)_{25^{-}}\right.$ PAMAM_E5.NSAH (dashed line). Recorded for the aqueous samples at dendrimer concentration of $1 \mathrm{mg} / \mathrm{mL}$ and $\mathrm{pH} 7.4$.

Figure 5s. Toxicity evaluation of $\mathbf{A}-$ PAMAM_E5. $\mathrm{NH}_{2}$ (solid line) and $\left\{\left(\mathrm{Ag}^{0}\right)_{2}\right.$ PAMAM_E5.NH 2$\}$ (doted line); B - PAMAM_E5.NGly (solid line) and C - $\left\{\left(\mathrm{Ag}^{0}\right)_{25^{-}}\right.$ PAMAM_E5.NGly $\}$ (doted line); PAMAM_E5.NSAH (solid line) and $\left\{\left(\mathrm{Ag}^{0}\right)_{25^{-}}\right.$ PAMAM_E5.NSAH $\}$ (doted line). The toxicity of DNCs and dendrimer templates is similar. Cytotoxicity of dendrimers and their silver nanocomposites was measured using XTT colorimetric assay of cellular viability. KB cells were incubated with nanocomposites at concentration range of 1 and $2000 \mathrm{nM}$ for $2 \mathrm{~h}$ at $37 \mathrm{C}$ in PBS buffer (pH 7.4). 
Figure 1s
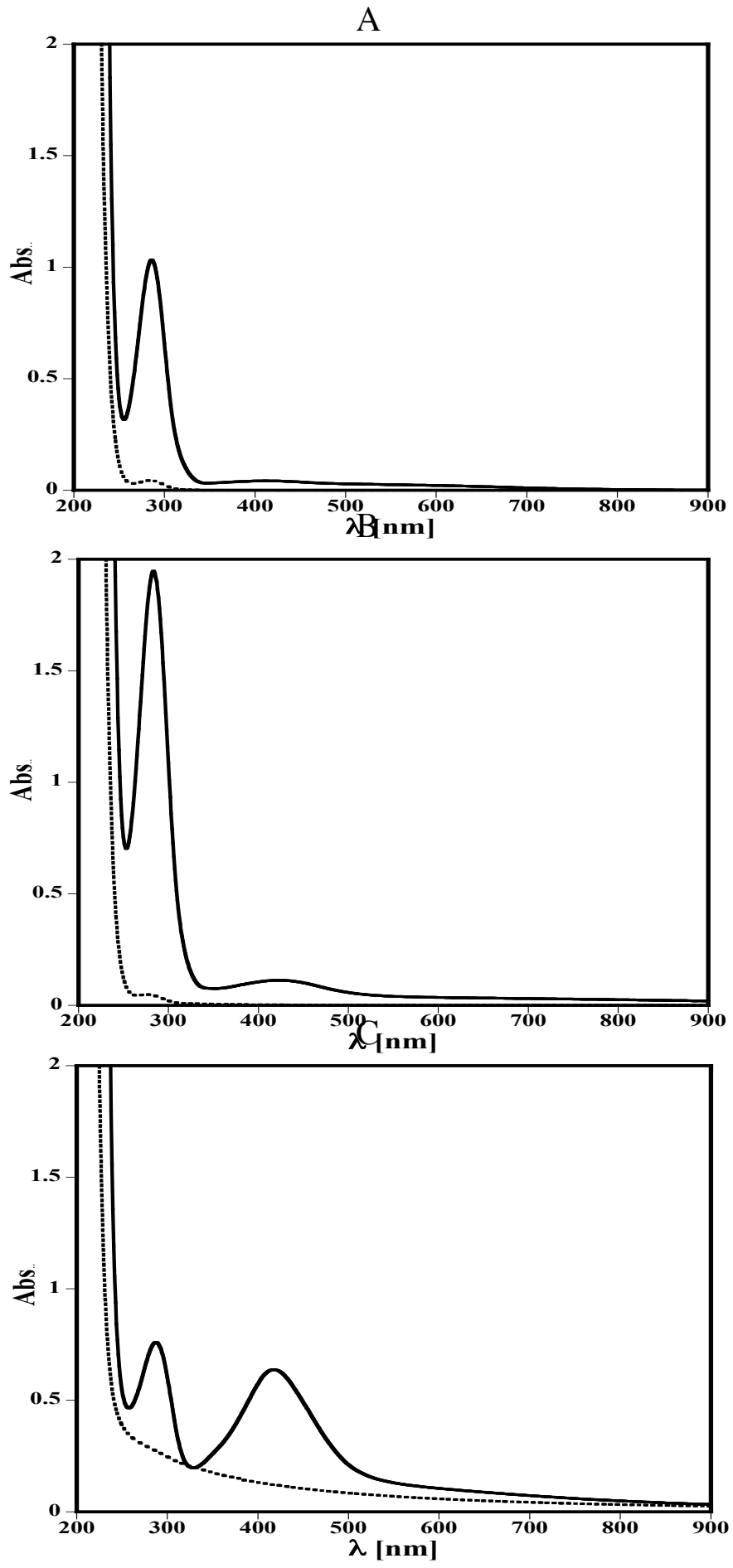

Lesniak et al

Figure 2s 
A

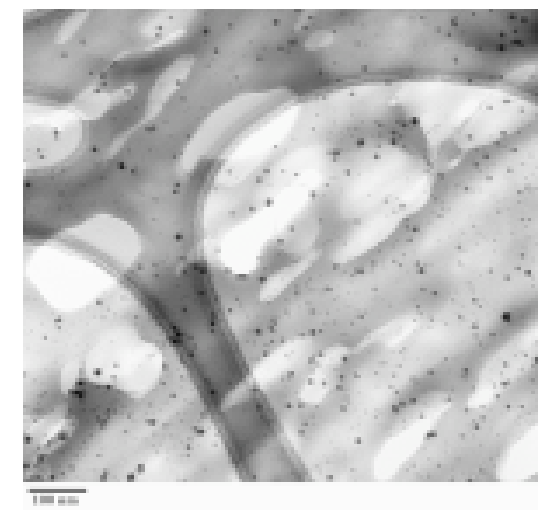

B

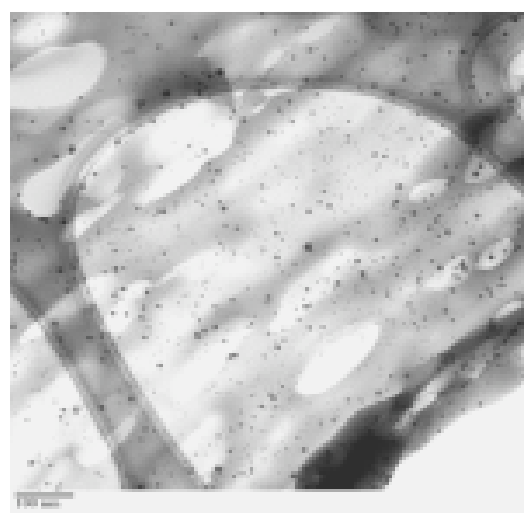

C

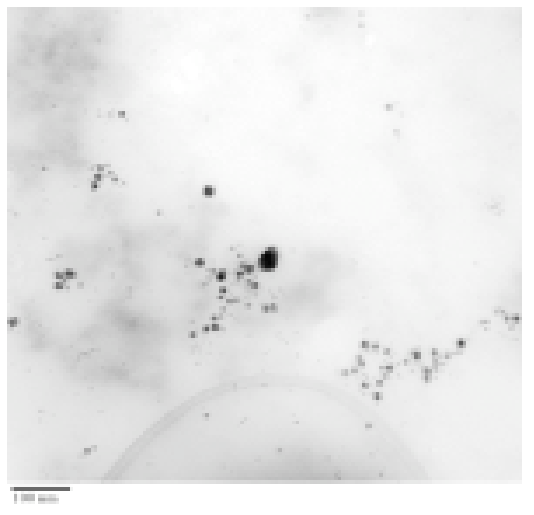

Lesniak at el 
Figure $3 \mathrm{~s}$

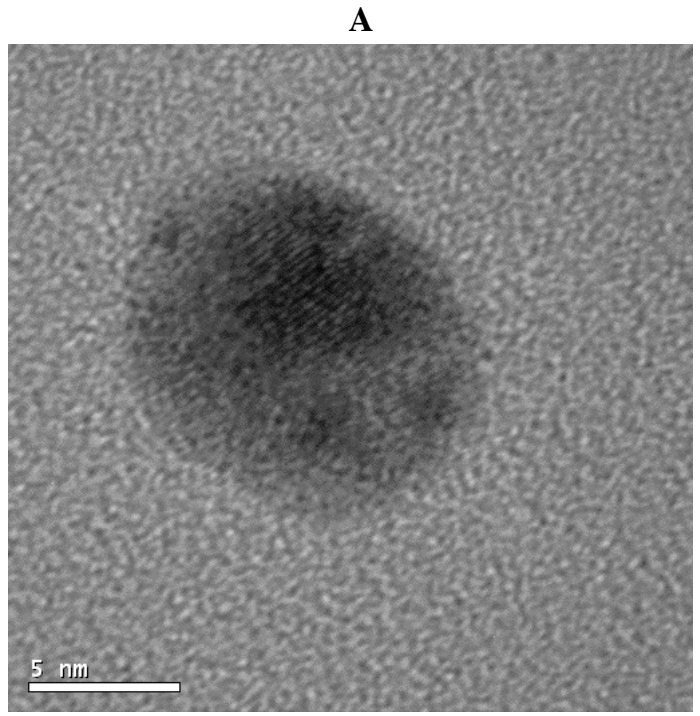

B
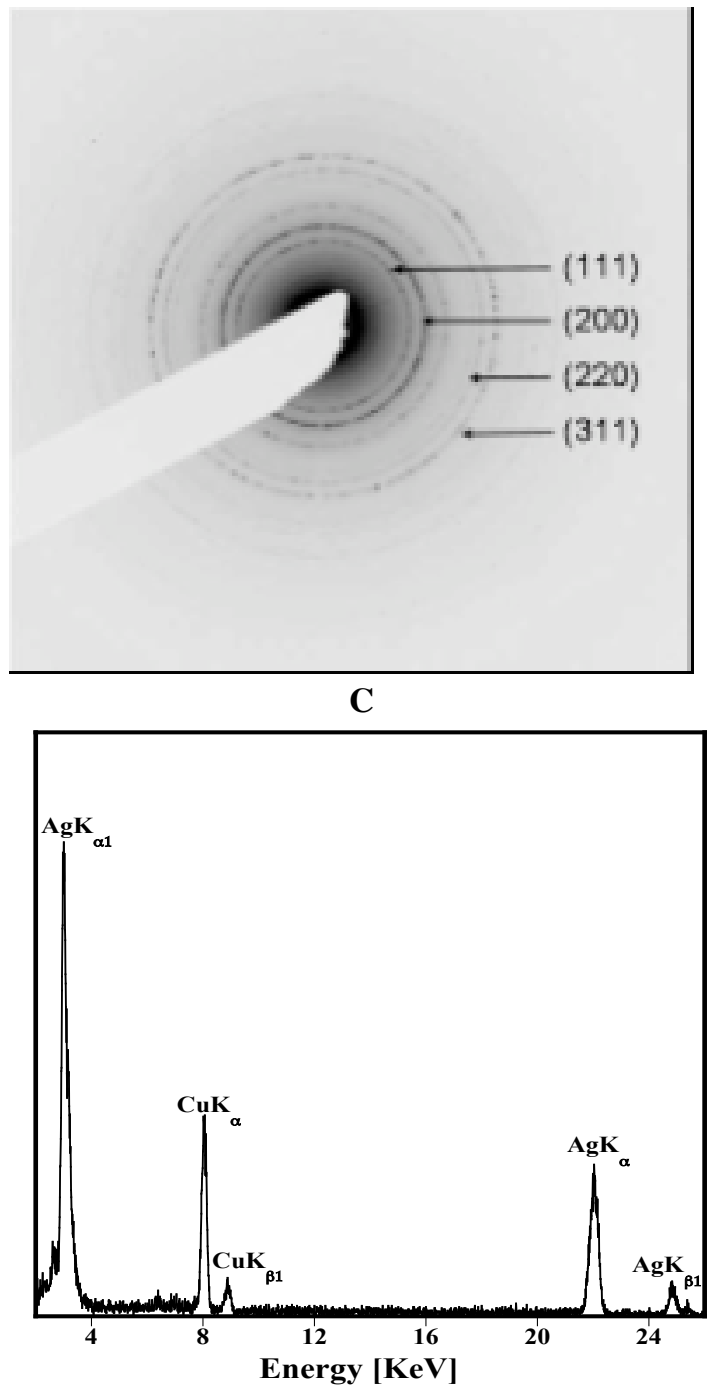

Lesniak et al 
Figure 4s
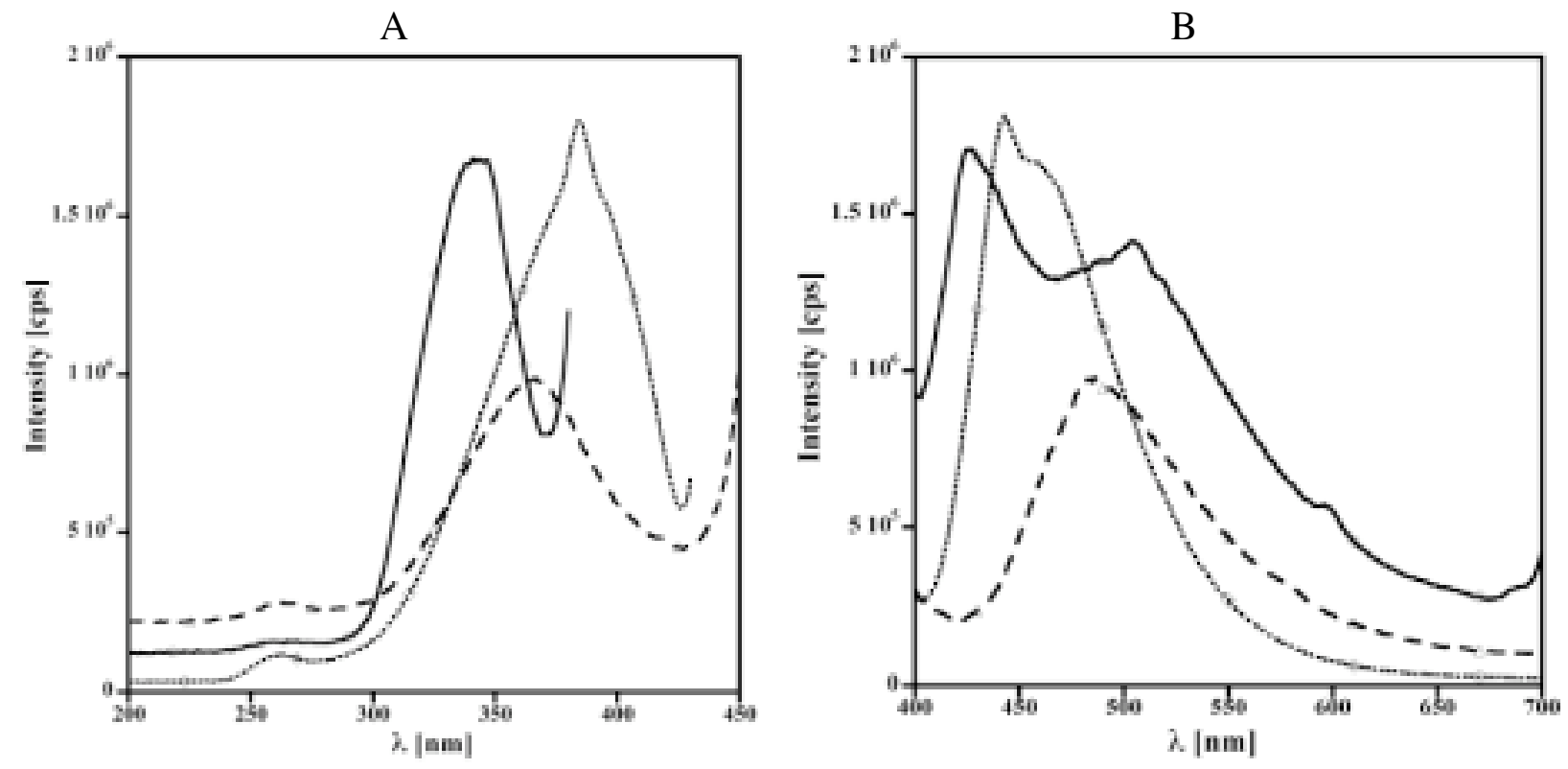

Lesniak et al

Figure 5s

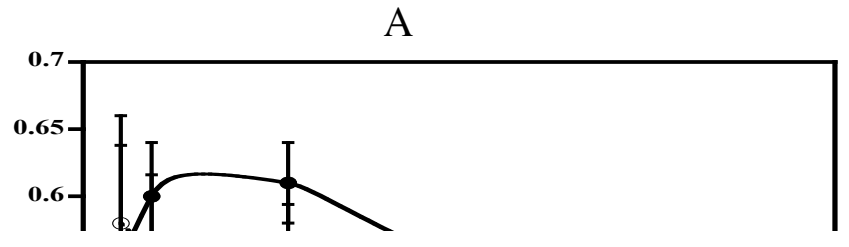


B
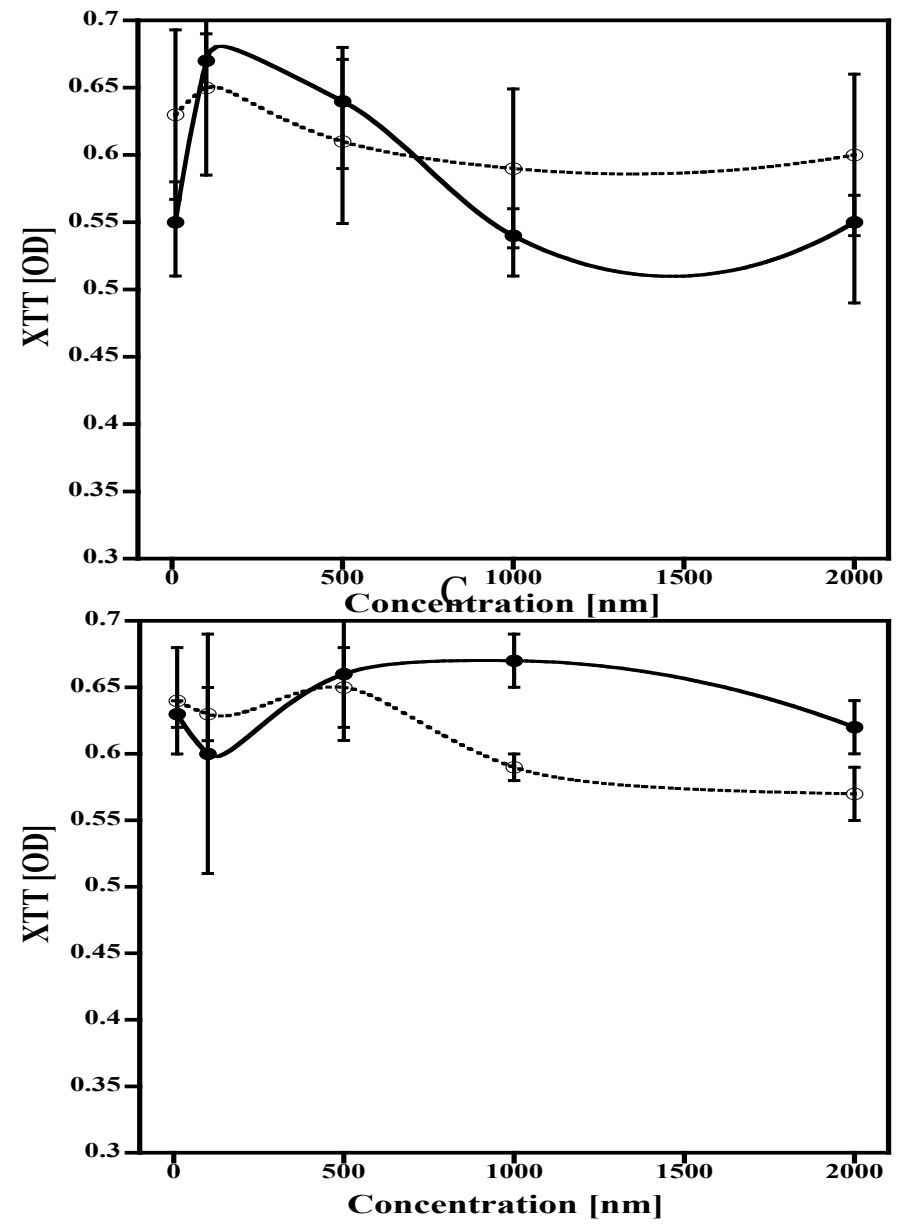

Lesniak et al 